\title{
Uso del recurso didáctico en el proceso enseñanza- aprendizaje en el periodo 2005-2012 en la LEBEM ${ }^{1}$
}

Using a teaching resource in the teaching-learning process in the period 2005-2012 in the LEBEM

Usando um recurso pedagógico no processo de ensinoaprendizagem no período de 2005-2012 no LEBEM

Recibido: diciembre de 2011

Aceptado: mayo de 2012
Angie Lorena Mendoza López ${ }^{2}$

Jhon Alexander Carrión Campo ${ }^{3}$

Juan Manuel Ariza Pedraza ${ }^{4}$

Josué Jaramillo Muñoz

\section{Resumen}

La comunicación propuesta pretende presentar una descripción de las fases por desarrollar en la fase I del proyecto de investigación "desarrollo de las prácticas en LEBEM en el periodo 2005-2012” del grupo de investigación CRISALIDA, adscrito a la Universidad Distrital, se presentaran los momentos en los que se desarrollara la investigación, la cual es de tipo documental y se basara en las unidades didácticas como instrumento de indagación y reflexión, específicamente este grupo de pasantes centrara la mirada en el énfasis de la práctica II, buscando evidenciar qué entendimientos y evoluciones se han presentado en torno a los recursos didácticos en el aula de matemáticas y su papel como herramienta de apoyo a procesos de enseñanza-aprendizaje.

Palabras clave: Recursos didácticos; investigación documental; unidades didácticas; enseñanza; metodología de enseñanza; análisis y reflexión sobre la enseñanza; sistematización.

\begin{abstract}
The proposed paper aims to present a description of the phases to be developed in Phase I of the research project "Development LEBEM practices in the period 2005-2012" CRISALIDA research group, attached to the University District, will present the moments in which research is developed, which is documentary and was based on the teaching units as an instrument of inquiry and reflection, this group of interns specifically focused look at the emphasis of practice II, seeking to show how understandings and developments have been filed about teaching resources in the mathematics classroom and its role as a tool to support teaching-learning processes.
\end{abstract}

1 Artículo de Investigación

2 Universidad Distrital Francisco José de Caldas. Bogotá, Colombia. Contacto: angie920811@hotmail.com

3 Universidad Distrital Francisco José de Caldas. Bogotá, Colombia. Contacto: jhonalexcc@hotmail.com

4 Universidad Distrital Francisco José de Caldas. Bogotá, Colombia. Contacto: mannuelthrash@hotmail.com

5 Universidad Distrital Francisco José de Caldas. Bogotá, Colombia. Contacto: jjaramillo3618@hotmail.com 
Keywords: Teaching resources, research documentary teaching units, teaching, teaching methodology, analysis and reflection on teaching; systematization.

\section{Resumo}

O trabalho proposto tem como objetivo apresentar uma descrição das fases a serem desenvolvidas na Fase I do projeto "práticas LEBEM desenvolvimento no período 2005-2012" pesquisa grupo de pesquisa CRISALIDA, ligado ao University District, apresentará os momentos em que se desenvolve a pesquisa, que é documentário e baseou-se nas unidades de ensino como instrumento de investigação e reflexão, este grupo de estagiários voltados especificamente olhada na ênfase da prática II, buscando mostrar como os entendimentos e os desenvolvimentos foram arquivados sobre recursos pedagógicos em sala de aula de matemática e de seu papel como uma ferramenta para apoiar os processos de ensino-aprendizagem.

Palavras-chave: Recursos de ensino, unidades de ensino documentais de pesquisa, ensino, metodologia de ensino, análise e reflexão sobre o ensino; sistematização.

\section{Introducción}

El presente proyecto de investigación surge debido a la necesidad de construir y reconstruir el proceso y el desarrollo de las prácticas docentes de los estudiantes para profesores de Matemáticas en el proyecto curricular LEBEM. Es necesario evidenciar la manera como se pone en juego el conocimiento en el aula por parte de los practicantes, ya que uno de los principios orientadores en LEBEM es formar un profesor crítico, reflexivo, investigador, constructivo, plural, diverso y complejo en el aula Lurduy (2000).

La práctica docente es concebida en el proyecto curricular LEBEM como "...como el conjunto de estrategias didácticas y mediaciones instrumentales que el profesor pone en juego en el aula para construir significados sobre los objetos matemáticos a partir de la interacción entre él y sus estudiantes o entre éstos." (Sánchez, Lurduy y Guerrero, 2005).

Los estudiantes de la LEBEM deberán dar cuenta de estrategias didácticas que permitan generar las condiciones necesarias para el aprendizaje matemático en el aula de educación básica, y que estas estrategias evidencien la relación de conceptos, teorías, medios de representación, materiales didácticos... etc., a través de las unidades didácticas.
En relación con lo anterior el presente grupo de pasantes, hará una investigación documental usando las unidades didácticas de la práctica intermedia II que ha sido realizadas en periodo 2005-2012 como un instrumento de indagación y análisis, la practica intermedia II tiene como énfasis el uso de los recursos didácticos, es decir que el análisis que será realizado por parte de los pasantes tendrá como eje central el uso de los recursos didácticos en el aula.

El grupo Crisálida para desarrollar esta investigación ha propuesto una investigación documental cualitativa de tipo descriptivo-exploratorio y se enmarca en los estudios de carácter histórico hermenéutico. Además hace uso de la técnica de análisis de contenido, este trabajo investigativo se basará en el análisis de exploración de contenido, el análisis de verificación de contenido y análisis de contenido cualitativo.

Entendiendo la investigación documental como el medio que permite analizar diferentes fenómenos de orden histórico, psicológico, social, entre otros, utilizando técnicas muy precisas, que permiten observar y reflexionar sistemáticamente sobre realidades teóricas o no.

La metodología que permite el desarrollo de una investigación documental parte de la elección del 
tema, la búsqueda de la bibliografía básica junto a las fichas bibliográficas, la lectura del material y con esto la delimitación del tema que permita establecer un esquema de trabajo, ampliando así el material sobre el tema ya delimitado, organizando las fichas de contenido y redactando el trabajo final como tal.

Una investigación documental se desarrolla a partir de una serie de fases, a continuación se presentará cada una de las fases y una breve descripción de las actividades que hasta el momento han sido realizadas en cada una de ellas:

- Preparatoria: En esta fase se realizaron una serie de capacitaciones con el fin de orientar el trabajo que se va a realizar, donde se destaca el seminario de capacitación sobre motivación e intereses investigativos y el seminario sobre el reconocimiento y apropiación del proyecto de investigación.

- Descripción: En esta fase se realizó la lectura de diversos documentos que sustentan la investigación y se procedió a recolectar los documentos "Unidades Didácticas"

- Interpretación: En esta fase se debe realizar una lectura extensiva (informativa) y luego una lectura intensiva (descriptiva) para iniciar el análisis de cada unidad didáctica y realizar las respectivas fichas bibliográficas, cabe mencionar que la investigación se encuentra en esta fase.

Se hace necesario mencionar que se concibe el análisis de contenido en un sentido amplio, como una técnica de interpretación de textos, ya sean escritos, grabados, pintados, filmados... etc. o cualquier forma diferente donde puedan existir toda clase de registros de datos, trascripción de entrevistas, discursos, protocolos de observación, documentos, videos,...etc. el denominador común de todos estos materiales es su capacidad para albergar un contenido que leído e interpretado adecuadamente nos abre las puertas al conocimientos que allí se encuentre. El análisis de contenido se basa en la lectura (textual o visual) como instrumento de recogida de información, lectura que a diferencia de la lectura común debe realizarse siguiendo el método científico, es decir, debe ser: sistemática, objetiva, replicable, y válida.

$\mathrm{Al}$ concebir que la lectura debe ser sistemática, se entiende la sistematización como un enfoque de investigación en educación que se ubica en las metodologías cualitativas del tipo Histórico - hermenéutica a partir de la técnica del análisis de contenido usando como fuentes o casos los informes de unidades didácticas de las prácticas docentes de los estudiantes para profesores de matemáticas del proyecto curricular LEBEM.

La sistematización de las unidades didácticas está siendo realizada utilizando diferentes redes categoriales, que son empleadas en el momento en el que se tienen las unidades recolectadas y se pasa a reducir la información. Esta reducción debe ser sustentada bajo

Tabla 1.

\begin{tabular}{|c|l|}
\hline Fase & \multicolumn{1}{|c|}{ Categoría } \\
\hline A & Estructura básica de la unidad didáctica. \\
\hline B & Énfasis de la práctica (recursos didácticos) \\
\hline C & Análisis de la unidad, de forma detallada \\
\hline D & Validación del análisis. \\
\hline
\end{tabular}

Fuente: Elaboración propia

diferentes criterios, en el momento se han establecido cuatro fases con diferentes criterios, las cuales son:

Fase A: corresponde a estructura básica de la unidad didáctica, se contemplan de forma superficial los aspectos constitutivos mínimos que debe tener la unidad didáctica, los cuales se considera que son: introducción, justificación, objetivos (general y específicos), marco teórico (matemático, metodológico, didáctico y político), apartado sobre recursos didácticos, ideograma, matriz de planeación, diseño de las actividades, protocolos, reflexión, conclusiones y bibliografía.

Fase B: corresponde al énfasis de la práctica (recursos didácticos), en la cual se examina de una manera un poco más detallada, la forma en la que aparecen 
los recursos didácticos respecto a los referentes teóricos, la clasificación de estos y particularmente en los diseños de las actividades, la hipótesis de aprendizaje en contraste con los resultados estipulados en los protocolos.

Fase C: corresponde al análisis de la unidad, de forma detallada, en esta fase, en análisis de la unidad se realizara bajo los criterios de: coherencia, cohesión, pertinencia y suficiencia, los cuales se contemplaran en cada una de las partes de la unidad, es decir que en cada apartado de la unidad de tendrá en cuenta aquellos criterios, buscando identificar que el texto es uniforme y que guarda relación en cada uno de los aspectos que aparecen estipulados en él.

Fase D: corresponde a la validación del análisis realizado por los pasantes, momento que será realizado bajo la asesoría de los docentes encargados de la dirección de la investigación, ya que es en esta fase se tienen en cuenta las fases anteriores y las categorías de análisis que han realizado los pasantes con el fin de cuantifican los elementos constitutivos de cada una de las unidades con una nota promedio de 1 a 5 , valor numérico que dará cuenta de la calidad de escritura y de argumentación del documento. Al tener las notas establecidas, se procederá a hacer un análisis estadístico de estas.

- Construcción teórica: En esta fase se pasará a realizar el informe al grupo de investigación.

- Extensión y publicación: En esta fase después de haber terminado y entregado el informe se aspira, poder mostrar el resultado a nivel nacional e internacional, dado que se considera que la información que se obtendrá con esta investigación, permitirá vislumbrar de una forma un poco más amplia la propuesta innovadora de la LEBEM y los resultados que ha obtenido a través del periodo de tiempo estipulado.

Para estudiantes que se encuentran en formación, el realizar una investigación que tenga como eje principal el análisis de las prácticas, ha permitido que se genere una reflexión personal, en torno a su propio desenvolvimiento como docente en el aula, ha permitido que se empiece a pensar la profesión docente desde la persona misma en contacto con un entorno $y$ un saber, y en particular a permitido evaluar la pertinencia del uso de los recursos didácticos, Godino (2002) sugiere en las distintas propuestas de reforma de curriculum matemático, el uso de materiales didácticos como un factor importante para mejorar la calidad de la enseñanza. Para lograr alguna reflexión en esta problemática, en su trabajo Uso de material tangible y gráfico-textual en el estudio de las matemáticas: superando algunas posiciones ingenuas presenta algunas reflexiones en torno a los siguientes aspectos: la noción y clasificación de material didáctico, funciones del material manipulativo y dos posiciones extremas respecto al uso de los instrumentos semióticos (formalismo y empirismo) -esto será mencionado al hablar de los instrumentos semióticos (recursos específicos). La forma en la que deben ser usados y los beneficios que estos brindan cuando son usados de forma correcta.

Algo que debe ser resaltado y que se tomó de este autor, es que el uso del material debe permitir el planteamiento de problemas significativos para los estudiantes, que puedan ser asumidos por ellos, apropiados a su nivel e intereses. El material en sí es inerte, tanto si es tangible como gráfico-textual. Por ende es importante tener en cuenta esto a lo largo de esta investigación: lo que se debe considerar como recurso didáctico no es el material concreto o visual, sino -en términos de Godino- la situación didáctica integral.

La importancia de la creación y el fortalecimiento de grupos de investigación como este, que evalúen en proceso de formación de los futuros docentes, recae en el hecho de que la investigación educativa con sus métodos, paradigmas y técnicas genere el conocimiento necesario para la renovación del pensamiento educativo.

Teniendo en cuenta que la complejidad de la educación radica en el simple hecho de que aquellos que en ella intervienen presentan por si mismos complejidades de acuerdo a su naturaleza social y humana, además, se desenvuelven en un contexto igualmente impreciso compuesto de condiciones 
sociales, políticas, económicas, culturales, entre otras, que tiene un efecto directo en la relación profesor-saber-estudiante.

\section{Referencias}

Godino, J. (2002). Uso de material tangible y gráfico-textual en el estudio de las matemáticas: superando algunas posiciones ingenuas. Portugal.

Guerrero, F y Sánchez, N (2002). Renovación curricular y formación del profesor de matemáticas para la educación básica: la practica pedagógica como eje articulador del conocimiento profesional del profesor.
Guerrero, F; Sánchez, N y Lurduy, O (2005). La práctica docente a partir de los Modelos DECA y TSD.

Guerrero, F (2009). Componente teórico para un modelo tutorial en práctica docente.

Guerrero, F \& Sánchez, N (2011). Descripción de la competencia cognitiva en práctica docente

Guerrero, F; Sánchez, N y Lurduy, O (2011). La práctica docente en el proyecto curricular LEBEM.

Lurduy (2000) Formación de profesores de matemáticas. 